\title{
Correction to: Automatic Synthesis Approach for Unconstrained Face Images Based on Generic 3D Shape Model
}

\author{
Hamid Ouanan $\mathbb{D}$, Mohammed Ouanan and Brahim Aksasse
}

\section{Correction to:}

Chapter "Automatic Synthesis Approach for Unconstrained Face Images Based on Generic 3D Shape Model" in:

V. Bhateja et al. (eds.), Embedded Systems and Artificial

Intelligence, Advances in Intelligent Systems and Computing 1076, https://doi.org/10.1007/978-981-15-0947-6_43

The original version of the book was inadvertently published with an incorrect affiliation of the co-authors of the chapter "Automatic Synthesis Approach for Unconstrained Face Images Based on Generic 3D Shape Model", which has now been corrected as the following:

M. Ouanan and B. Aksasse

Department of Computer Science, M2I Laboratory, Faculty of Science and Techniques, Moulay Ismail University, BP 509 Boutalamine 52000 Errachidia, Morocco

The chapter and book have been updated with the changes. 